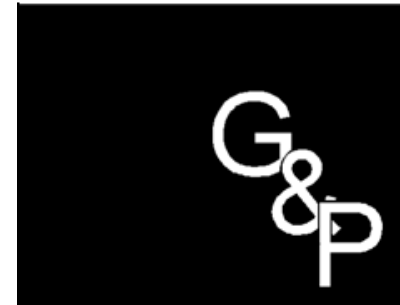

GESTÃO

\&

PRODUÇÃO

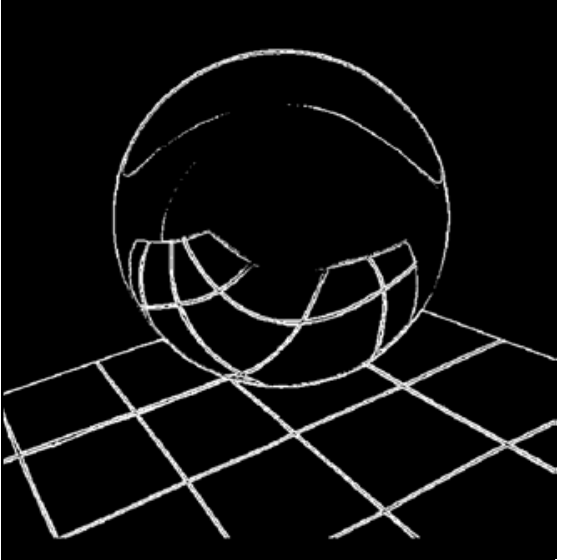

v.6, n.1, p. 61-76, abr. 1999

\section{UMA METODOLOGIA DE ANÁLISE ESTRATÉGICA DA TECNOLOGIA}

Marcelo Caldeira Pedroso

Departamento de Engenharia de Produção

Escola Politécnica

Universidade de São Paulo

\title{
Resumo
}

O presente trabalho propõe uma metodologia de análise estratégica da tecnologia nas empresas. Para tanto, ele apresenta uma conceituação e uma classificação de tecnologia, bem como adota um conjunto de critérios competitivos desdobrados da manufatura. A partir destas questões, é apresentada uma metodologia de análise estratégica da tecnologia que é estruturada na inter-relação entre os tipos de tecnologia e os critérios competitivos da manufatura adotados. A aplicação da metodologia proposta considera as abordagens 'technological-push' e 'marketing-pull' como impulsionadoras da decisão da adoção de novas tecnologias. Ao final, o trabalho apresenta um exemplo de aplicação que utiliza a teoria dos conjuntos difusos ('fuzzy set theory'), bem como algumas conclusões sobre a metodologia proposta.

Palavras-chave: estratégia de manufatura; tecnologia; análise estratégica da tecnologia; teoria dos conjuntos difusos.

\section{Introdução}

A adoção de novas tecnologias é uma questão fundamental para as empresas. Sob a ótica da competitividade, esta decisão pode contribuir fortemente para a melhoria do desempenho das empresas (PEGELS \& THIRUMURTHY, 1996); ao contrário, a relutância em introduzir novas tecnologias pode deteriorar o desempenho de uma determinada 
empresa em relação aos seus concorrentes (CHEN \& SMALL, 1996). Assim, a decisão referente à implantação de novas tecnologias é, por natureza, uma decisão estratégica uma vez que:

- ela afeta o desempenho e a competitividade da empresa;

- este tipo de decisão é geralmente difícil de se reverter, seja em função do volume de investimentos envolvidos como também pelos esforços organizacionais exigidos; e

- a introdução de uma nova tecnologia representa uma descontinuidade em relação à tecnologia atual, o que implica na necessidade de gerenciar a mudança - e, em alguns casos, esta questão pode ser mais importante até do que a própria tecnologia.

Diversos trabalhos propõem metodologias para integrar a decisão da adoção de novas tecnologias à estratégia das empresas (KLEINDORFER \& PARTOVI, 1990; MITCHELL, 1990; MADU \& GEORGANTZAS, 1991; NAIK \& CHAKRAVARTY, 1992; BETZ, 1993; GEHANI, 1995; KUEI et al., 1995; CHEN \& SMALL, 1996; KAKATI, 1997). Neste contexto, este trabalho apresenta uma metodologia que objetiva suportar a análise de novas tecnologias, cuja aplicação se insere no processo de desenvolvimento da estratégia de manufatura das empresas. O desenvolvimento desta metodologia é baseado em três questões:

- a conceituação da tecnologia;

- os critérios competitivos da manufatura; e

- as abordagens seguidas na decisão da adoção de novas tecnologias.

A partir da discussão de cada uma destas questões, este trabalho apresenta uma metodologia de análise estratégica da tecnologia e um exemplo de aplicação.

\section{A Conceituação de Tecnologia}

$\mathrm{O}$ termo tecnologia é amplamente difundido nos meios acadêmicos, governamentais e no ambiente das empresas; porém seu significado pode variar em função do contexto em que ele é utilizado. Este trabalho adota a conceituação de tecnologia segundo três considerações:

- os componentes da tecnologia, que contemplam três questões fundamentais (STONEBRAKER \& LEONG, 1994):

- a pesquisa, que diz respeito à descoberta de novos e abstratos conhecimentos;

- o desenvolvimento, que considera a aplicação prática do conhecimento; e

- a mudança, que aborda a utilização efetiva do conhecimento, substituindo ou complementando o conhecimento anterior.

- o nível de análise da tecnologia, que considera o estudo desta segundo três níveis de abrangência:

- o universo macro, que considera o campo de estudo delimitado segundo características geográficas, comerciais ou políticas em diferentes níveis de agregação. Como exemplo, pode-se citar a análise do desenvolvimento da tecnologia segundo blocos de países: Mercosul, Alca, Mercado Comum Europeu, Tigres Asiáticos, Países Emergentes, etc.; ou de nações: Japão, Alemanha, EUA, Brasil, etc.; ou de regiões: a região Sudeste do Brasil, a Nova Inglaterra nos EUA, etc. (alguns exemplos podem ser encontrados em Nelson, 1993; GASPARIKOVA, 1995; e TONG \& ZHAO, 1995);

- o universo meso, que analisa a tecnologia no âmbito de setores industriais - por exemplo: a indústria química (ASHSYERI et al., 1996), a indústria de bens de capital (KOHLER \& SCHMIERI, 1991), a indústria de marcapassos cardíacos (HIDEFJALL, 1995), o setor de biotecnologia (JOLLY \& RAMANI, 1995), o setor de telecomunicações (REAVILL \& KAMALL, 1995), etc. - ou das redes de suprimentos - por exemplo: as redes de suprimentos da indústria automotiva (LEE \& OAKES, 1995), da indústria aeroespacial (SHAW, 1995), da indústria de computadores pessoais (BERRY \& NAIM, 1996), da indústria de combustíveis (BOWER \& YOUNG, 1995), etc.; e 
- o universo micro, que estuda a tecnologia no contexto das empresas - por exemplo: a gestão estratégica da tecnologia na Sony, na NEC e na GE (BETZ, 1993); as alianças tecnológicas Vallée-IHC, ValléeVetcorp (VASCONCELLOS \& WAACK, 1995) e HP-Intel (JOLLY \& HUMBERT, 1995), etc.

- a classificação da tecnologia, que contempla o desdobramento desta em cinco tipos - ou categorias (STONEBRAKER \& LEONG, 1994):

- tecnologia de processo, que aborda a pesquisa, o desenvolvimento e a introdução de novas tecnologias de processo (ex.: a utilização de moldes de espuma em substituição aos moldes metálicos no processo de fundição de motores na fábrica 'Saturn' da 'General Motors');

- tecnologia de materiais, que considera a pesquisa, o desenvolvimento e a introdução de novos materiais (ex.: a utilização de materiais desenvolvidos para aplicações espaciais, tais como o grafite e alguns tipos de plástico, na fabricação de bicicletas);

- tecnologia de produtos e serviços, que contempla a pesquisa, o desenvolvimento e a introdução de novos produtos e serviços (ex.: em relação a novos produtos, o desenvolvimento de novos processadores - ou 'chips' - na indústria de computadores; quanto aos novos serviços, o conceito de bancos virtuais e a utilização dos serviços bancários pela 'internet');

- tecnologia de informação, que diz respeito à pesquisa, ao desenvolvimento e à introdução de novas tecnologias de informação (ex.: a utilização de EDI's de maneira integrada aos Sistemas ERP);

- tecnologia de gestão, que considera a pesquisa, o desenvolvimento e a introdução de novas técnicas de gestão. Na realidade atual, podem ser citados a implantação do Sistema da Qualidade QS 9000, a aplicação do conceito de ECR - ou
'Efficient Consumer Response' -, a implantação dos denominados Sistemas de PPCP com Capacidade Finita (PEDROSO \& CORRÊA, 1996) (ou FCS - 'Finite Capacity System', também denominados recentemente de APS - 'Advanced Planning Systems'), bem como a utilização de sistemas de suporte à decisão para planejamento de redes de suprimentos.

Em síntese, o presente trabalho adota a definição da tecnologia segundo os três componentes fundamentais - a pesquisa, o desenvolvimento e a mudança - e os cinco tipos de tecnologia - de processo, de materiais, de produtos e serviços, de informação e de gestão - dentro do universo de análise micro, ou seja, no âmbito das empresas. A Figura 1 delimita a conceituação de tecnologia no escopo deste trabalho.

\section{Os Critérios Competitivos da Manufatura}

耳 ste trabalho adota os cinco critérios SLACK (1993). Esta decisão está baseada no fato deste conjunto de critérios abranger a maioria dos citados pela literatura no âmbito da manufatura (PEDROSO, 1996). Os critérios adotados são:

- qualidade, que diz respeito à capacidade da empresa identificar as necessidades e expectativas dos clientes, transformá-las em especificações do produto e atender estas especificações;

- velocidade, que considera a rapidez com que a empresa entrega o produto a partir da solicitação por parte do cliente;

- pontualidade, que contempla o cumprimento das datas de entrega acordadas com o cliente;

- flexibilidade, que aborda a habilidade do sistema de manufatura em se adaptar com eficácia e eficiência às mudanças não-planejadas nos seus ambientes interno e externo; e

- custo, que considera o volume de recursos financeiros envolvidos na manufatura.

A Figura 2 ilustra os critérios competitivos da manufatura adotados neste trabalho. 


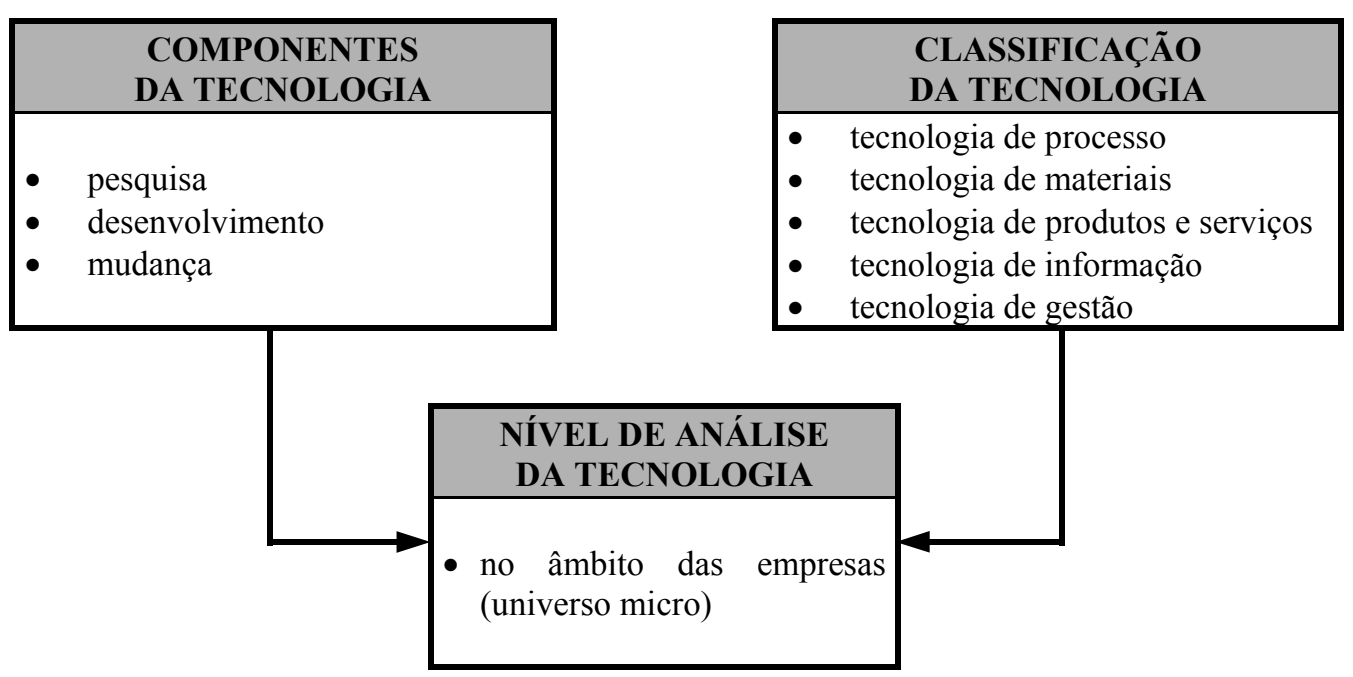

Figura 1 - A conceituação de tecnologia no escopo deste trabalho.

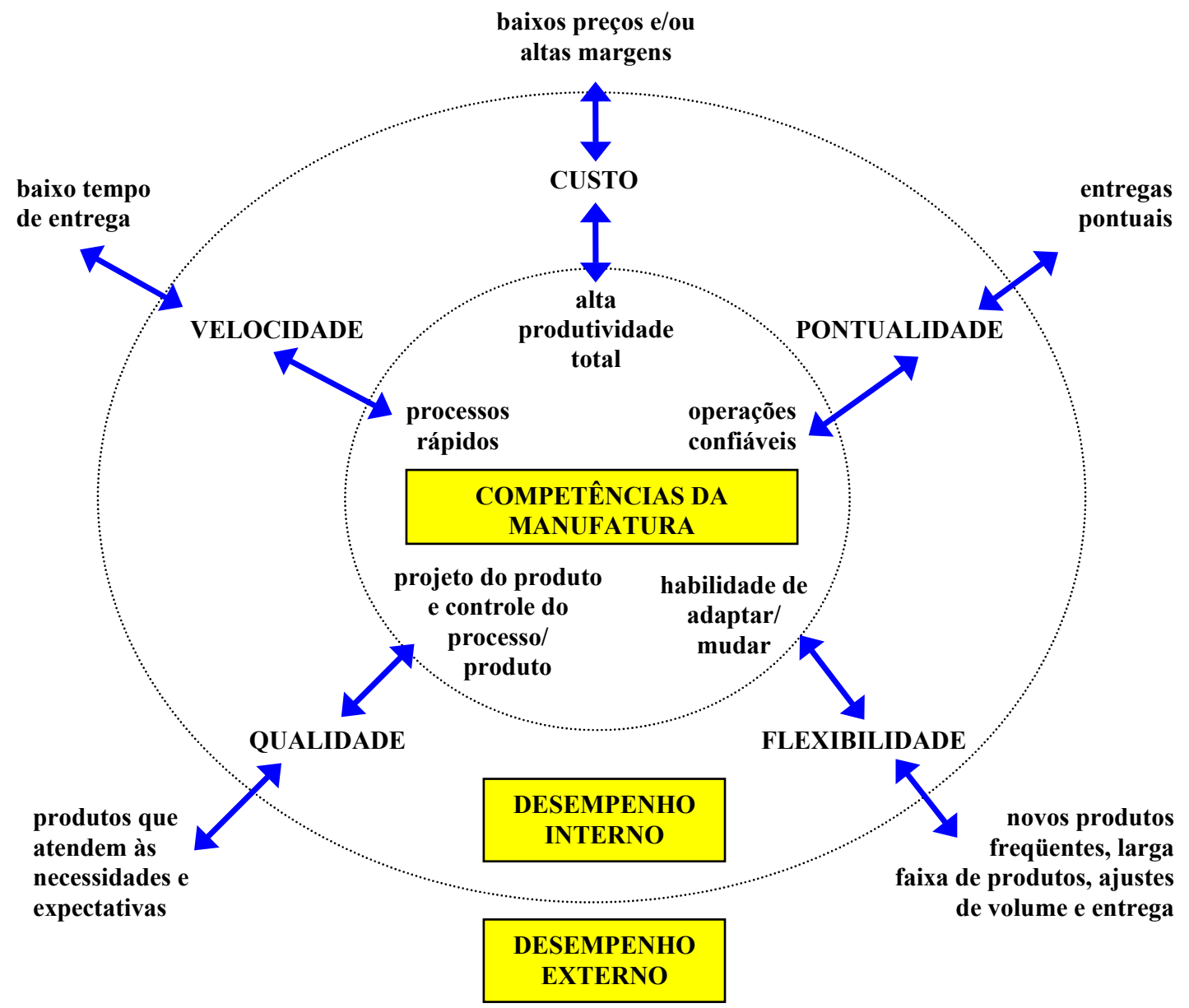

Figura 2 - Os critérios competitivos da manufatura (adaptado de SLACK, 1993). 


\section{Quadro 1 - O conjunto de critérios competitivos desdobrados da manufatura utilizado por PEDROSO (1996).}

\begin{tabular}{|c|c|c|}
\hline $\begin{array}{c}\text { CRITÉRIO } \\
\text { COMPETITIVO }\end{array}$ & DESDOBRAMENTO & DEFINIÇÃO \\
\hline \multirow{8}{*}{ QUALIDADE } & Desempenho funcional & São as principais características funcionais de um produto. \\
\hline & Características adicionais & São as características que complementam as funções básicas de um produto. \\
\hline & Confiabilidade & É a probabilidade de um produto falhar em um determinado período. \\
\hline & Conformidade & $\begin{array}{l}\text { É o grau em que o projeto de um produto e respectivas características funcionais } \\
\text { e adicionais atendem padrões preestabelecidos. }\end{array}$ \\
\hline & Durabilidade & É o tempo de vida de um produto até a sua substituição. \\
\hline & Manutenibilidade & $\begin{array}{l}\text { É a velocidade, cortesia, competência e facilidade de manutenção de um produto } \\
\text { uma vez que este tenha falhado. }\end{array}$ \\
\hline & Estética & $\begin{array}{l}\text { É a forma como um produto é julgado pelos sentidos humanos (visão, paladar, } \\
\text { audição, tato e olfato). }\end{array}$ \\
\hline & Qualidade percebida & $\begin{array}{l}\text { É a imagem concebida pelo consumidor em relação aos atributos de um produto } \\
\text { quando não se tem informações completas a respeito deste. }\end{array}$ \\
\hline \multirow{4}{*}{ VELOCIDADE } & Desenvolvimento & É o tempo de desenvolvimento do produto e do processo. \\
\hline & Aquisição & É o tempo de aquisição e recebimento das matérias-primas. \\
\hline & Produção & É o tempo de fabricação e montagem dos produtos. \\
\hline & Entrega & É o tempo de processamento dos pedidos dos clientes e de distribuição dos produtos. \\
\hline \multirow{2}{*}{$\begin{array}{l}\text { PONTUA- } \\
\text { LIDADE }\end{array}$} & $\begin{array}{l}\text { Realismo ao prometer } \\
\text { prazos }\end{array}$ & É a diferença entre a data de entrega prometida e a programada. \\
\hline & $\begin{array}{l}\text { Pontualidade ao atender os } \\
\text { prazos }\end{array}$ & É a diferença entre a data de entrega programada e a real. \\
\hline \multirow{10}{*}{ FLEXIBILIDADE } & Novos produtos (faixa) & $\begin{array}{l}\text { São as quantidades possíveis de introdução de novos produtos ou de alteração } \\
\text { dos produtos existentes. }\end{array}$ \\
\hline & Novos produtos (resposta) & É o tempo necessário para a introdução de novos produtos ou alteração dos existentes. \\
\hline & 'Mix' de produtos (faixa) & São os limites possíveis para variação do 'mix' de produtos. \\
\hline & 'Mix' de produtos (resposta) & É o tempo necessário para ajustar o 'mix' de produtos. \\
\hline & Volume (faixa) & São os limites possíveis para alteração nos níveis agregados de produção. \\
\hline & Volume (resposta) & É o tempo necessário para alterar os níveis agregados de produção. \\
\hline & Entrega (faixa) & É a variação possível quanto à alteração das datas de entrega prometidas. \\
\hline & Entrega (resposta) & É o tempo necessário para alterar as datas de entrega prometidas. \\
\hline & Robustez (faixa) & $\begin{array}{l}\text { São as alternativas possíveis para responder aos problemas decorrentes de mudan- } \\
\text { ças não-planejadas na disponibilidade dos recursos e no suprimento dos insumos. }\end{array}$ \\
\hline & Robustez (resposta) & $\begin{array}{l}\text { É o tempo necessário para responder aos problemas decorrentes de mudanças } \\
\text { não-planejadas na disponibilidade dos recursos e no suprimento dos insumos. }\end{array}$ \\
\hline \multirow{4}{*}{ CUSTO } & Custo do produto & $\begin{array}{l}\text { São os custos associados às atividades de suporte à instalação industrial, de su- } \\
\text { porte ao produto, ao nível do lote de produção e ao nível da unidade produzida. }\end{array}$ \\
\hline & Utilização da capacidade & $\begin{array}{l}\text { É a medida de ocupação da capacidade, indicando os custos associados à } \\
\text { manutenção de capacidade superior à demanda. }\end{array}$ \\
\hline & $\begin{array}{l}\begin{array}{l}\text { Produtividade do } \\
\text { investimento fixo }\end{array} \\
\end{array}$ & $\begin{array}{l}\text { É a medida do nível de investimento alocado à tecnologia de processo da } \\
\text { empresa visando obter um determinado nível de capacidade. }\end{array}$ \\
\hline & $\begin{array}{l}\text { Capital alocado aos } \\
\text { estoques }\end{array}$ & $\begin{array}{l}\text { É a medida do valor monetário associado à decisão de manter estoques (matérias- } \\
\text { primas, em processo e produtos acabados). }\end{array}$ \\
\hline
\end{tabular}

Por outro lado, este conjunto de critérios apresenta um caráter multidimensional e, desta forma, devem ser desdobrados em categorias mais específicas para uma análise mais adequada (GARVIN, 1993). Assim, este trabalho adota o desdobramento utilizado por PEDROSO (1996), conforme apresentado no Quadro 1.
Deve-se ainda considerar que a escolha do conjunto de critérios competitivos da manufatura, bem como o respectivo desdobramento, deve ser aderente à realidade da empresa analisada. Assim, uma aplicação prática deve contemplar a possibilidade de exclusão de determinados critérios - que eventualmente não mereçam ser 
considerados - e a inclusão de critérios competitivos e/ou de desdobramentos adicionais - caso os critérios previamente adotados não contemplem, de maneira satisfatória, todos aqueles que devam ser analisados.

\section{As Diferentes Abordagens na Decisão da Adoção de Novas Tecnologias}

A decisão referente à adoção de uma nova tecnologia na empresa pode ser originada basicamente em dois "pontos de origem" (GEHANI, 1995) - ou "fatores motivacionais" (MUNRO \& NOORI, 1988) -, que são as bases das abordagens:

- 'technological-push', que se origina no reconhecimento de como uma nova tecnologia pode prover uma melhoria no desempenho da empresa (MUNRO \& NOORI, 1988). Esta abordagem enfoca a ótica do fornecedor como ponto de origem da adoção da nova tecnologia (GEHANI, 1995), ou seja, a visão do provedor ou do usuário desta; e

- 'marketing-pull', que considera as necessidades do mercado e dos clientes como ponto de origem (GEHANI, 1995). Estas necessidades são originadas na identificação de deficiências do desempenho da empresa ou na percepção de novas oportunidades que podem, em ambos os casos, ser exploradas a partir da adoção de novas tecnologias (MUNRO \& NOORI, 1988).

Neste contexto, a postura gerencial proativa está associada à identificação de oportunidades tanto de mercado como de novas tecnologias na decisão da adoção de novas tecnologias. Ao contrário, a postura reativa está relacionada à identificação de deficiências no desempenho da empresa como fator motivador para a introdução de novas tecnologias (MUNRO \& NOORI, 1988).

A Figura 3 resume os conceitos discutidos na decisão da adoção de novas tecnologias por parte das empresas.

A consideração destas abordagens nas empresas é uma questão contingencial, que depende de fatores internos - por exemplo, a cultura gerencial da empresa - e externos - por exemplo, o quão dinâmico e competitivo é o mercado onde a empresa atua. MUNRO \& NOORI (1988) pressupõem a utilização das duas abordagens - 'marketing-pull' e 'technologicalpush' - simultaneamente como uma boa prática; eles denominam este processo de integração 'push-pull' visando caracterizar o balanceamento destes dois fatores motivacionais na decisão da adoção de novas tecnologias.

\section{Descrição da Metodologia de Análise Estratégica da Tecnologia Proposta}

A metodologia de análise estratégica da tecnologia proposta é estruturada na interrelação entre os tipos de tecnologia e o conjunto de critérios competitivos da manufatura adotado. A Figura 4 ilustra, de maneira genérica, este conceito.

Basicamente, esta metodologia objetiva identificar o impacto de cada tecnologia específica nos critérios competitivos da manufatura. Por outro lado, as relações entre estes critérios e os tipos de tecnologia podem não ser necessariamente diretas. Por exemplo, a implantação de um Sistema MRP-II contempla dois tipos de tecnologia: a tecnologia de gestão que está relacionada à utilização deste sistema para apoiar as decisões no âmbito dos sistemas de PPCP, bem como de algumas boas práticas para o gerenciamento da manufatura, tais como o inventário rotativo, a avaliação de fornecedores, etc. - e a tecnologia de informação - que contempla a integração das informações entre as funções da empresa. Neste exemplo, o impacto desta tecnologia da informação nos critérios competitivos da manufatura não é totalmente evidente. Desta forma, propõe-se utilizar uma relação indireta, pela associação da tecnologia aos processos de negócios - ou às áreas de decisão da manufatura (que segmentam a manufatura em áreas com conjuntos delimitados de decisões) - da empresa e estes aos critérios competitivos da manufatura. Esta associação 


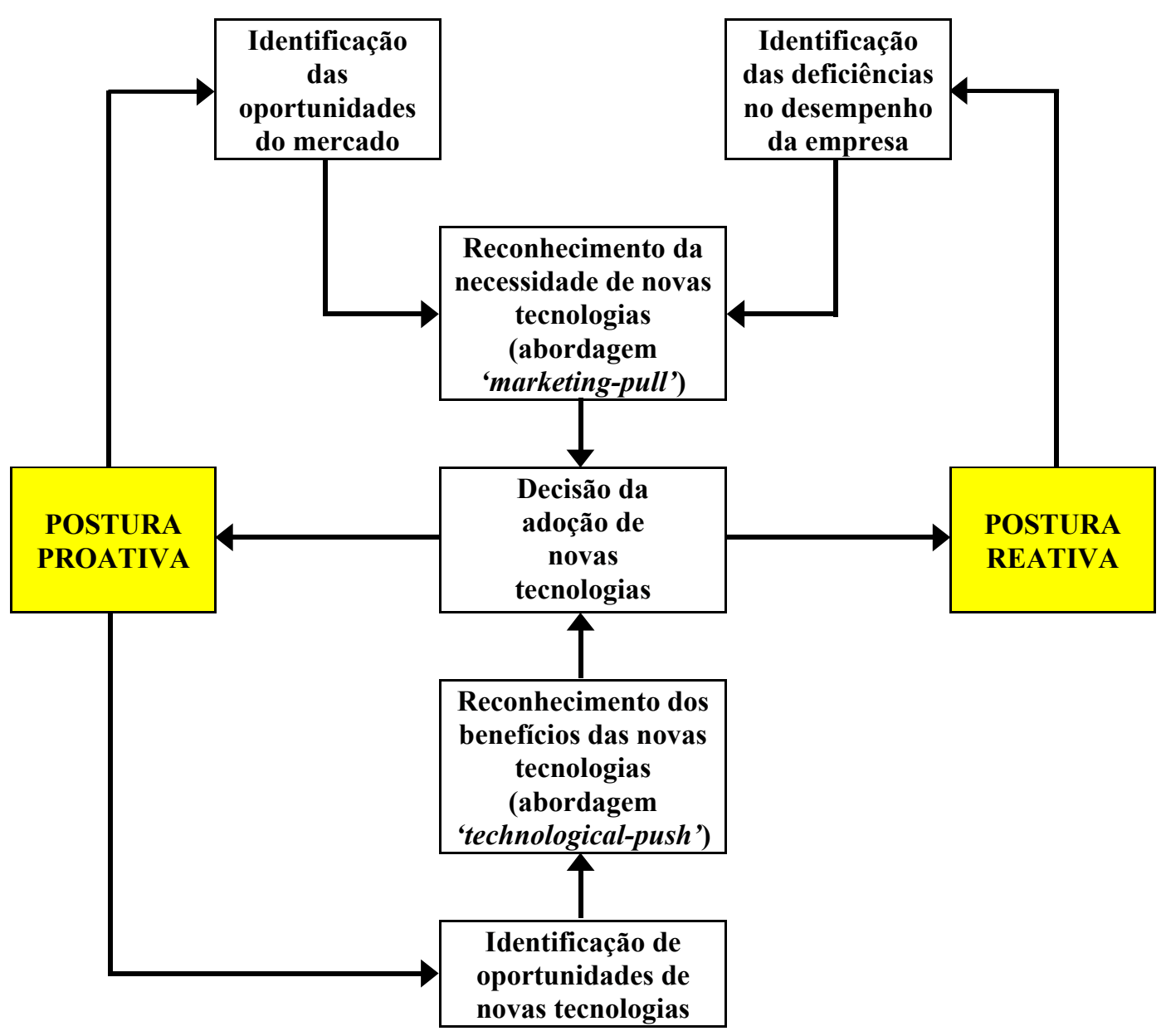

Figura 3 - As abordagens proativa e reativa na decisão da adoção de novas tecnologias (adaptado de MUNRO \& NOORI, 1988).
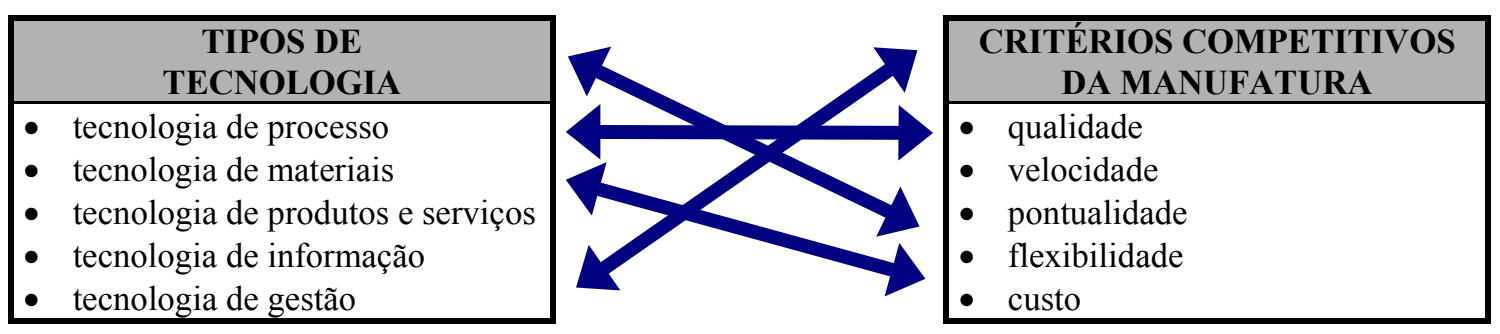

Figura 4 - A Inter-Relação Genérica Entre os Tipos de Tecnologia e os Critérios Competitivos da Manufatura.

também é utilizada na metodologia prescrita por KEINDORFER \& PARTOVI (1990), que aborda a integração da escolha da tecnologia à estratégia de manufatura. Assim, por esta associação, é possível mapear o impacto da tecnologia nestes critérios ou vice-versa, ou seja, como cada critério é atendido mediante uma determinada tecnologia. A Figura 5 ilustra esta associação por meio de matrizes de inter-relação, que foram baseadas nas matrizes utilizadas na 


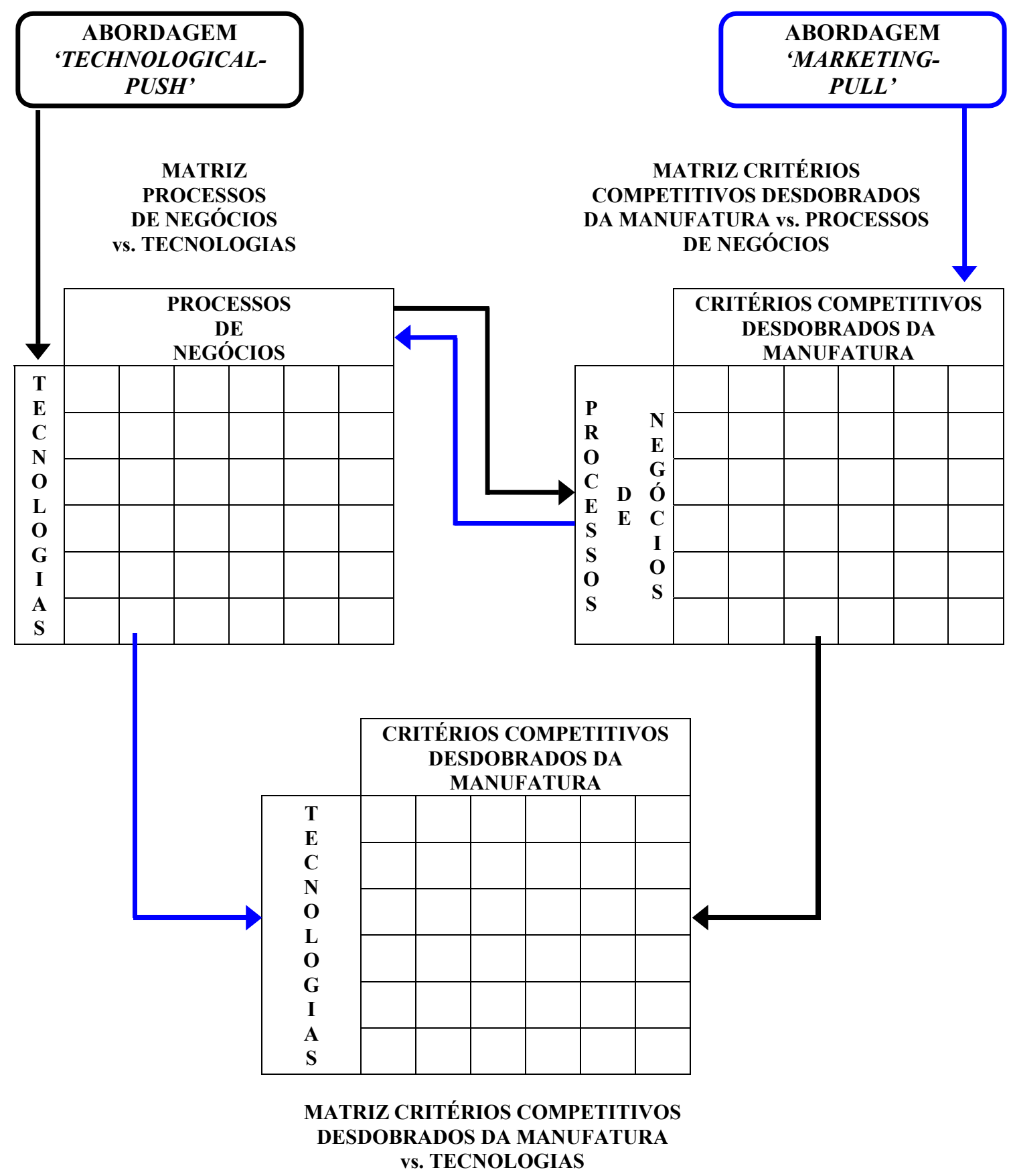

Figura 5 - As matrizes de inter-relações tecnologias vs. processos de negócios vs. critérios competitivos desdobrados da manufatura.

metodologia de avaliação da aquisição de novas tecnologias de manufatura, proposta por NAIK \& CHAKRAVARTY (1992).

Deve-se considerar que, como o processo de mapeamento é biunívoco, a análise correspon- dente pode ser originada tanto da tecnologia específica como dos critérios competitivos da manufatura (Figura 5). Assim, esta metodologia pode abordar tanto a abordagem 'marketingpull' - que contempla a origem da análise nos 


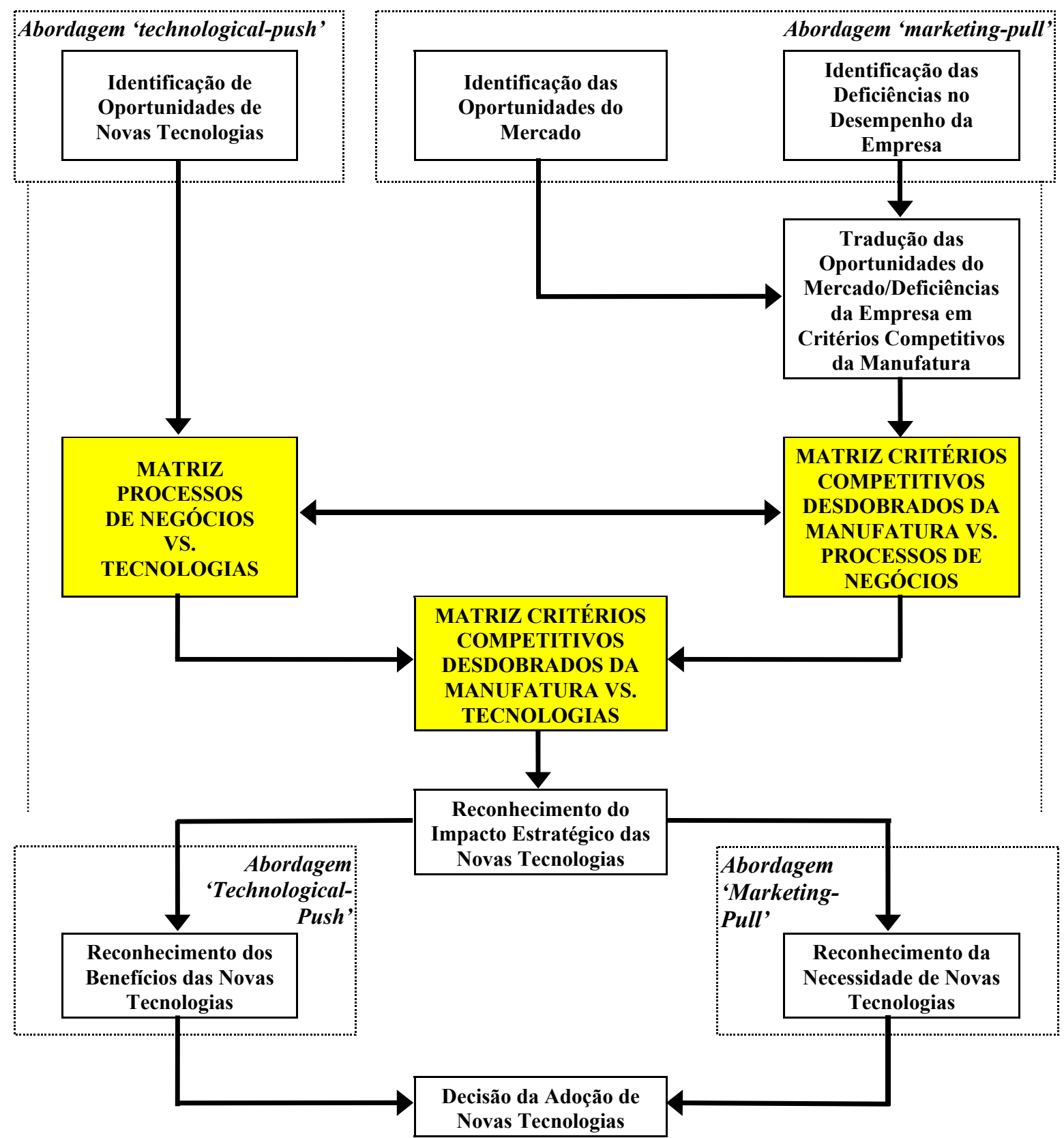

Figura 6 - Metodologia de análise estratégica da tecnologia.

critérios competitivos da manufatura - quanto a abordagem 'technological-push' - que considera a análise a partir da tecnologia específica. A Figura 6 ilustra a metodologia proposta neste contexto.

Diferentes métodos de inter-relacionamento entre as variáveis critérios competitivos, processos de negócios (ou áreas de decisão) e tecnologias podem ser utilizados, tais como: o método AHP ('analytical hierarchical process') (KLEINDORFER \& PARTOVI, 1990; ROPERLOWE \& SHARP, 1990; MADU \& GEORGANTZAS, 1991; MOHANTY \& VENKATARAMAN， 1993; O'BRIEN \& 
SMITH, 1993; KUEI et al., 1994; ALBAYRAKOGLU, 1996), a técnica do QFD ('quality function deployment') (CROWE \& CHENG, 1996), as matrizes de medidas de desempenho (NOORI \& GILLEN, 1995), o modelo de índices compostos por múltiplos critérios ('multiple-criteria composite index model') proposto por COOK \& KRESS (1994) e o procedimento de inter-relação de índices qualitativos sugerido por NAIK \& CHAKRAVARTY (1992). Por outro lado, alguns critérios também podem ser adotados para analisar a adoção de novas tecnologias, tais como: a aceitabilidade, a viabilidade e a vulnerabilidade das possíveis alternativas tecnológicas (SLACK, 1993); ou o potencial de melhoria, a adequação às competências atuais da manufatura e a facilidade de implantação (GARVIN, 1993).

Em síntese, a metodologia de análise estratégica da tecnologia proposta sugere a utilização de matrizes de inter-relacionamento das variáveis critérios competitivos da manufatura, processos de negócios e tecnologias visando analisar o impacto estratégico de novas tecnologias. Esta análise pode ser originada pela identificação de oportunidades de novas tecnologias, da identificação das oportunidades do mercado e das deficiências no desempenho da empresa. O resultado da aplicação da metodologia proposta é a avaliação do impacto estratégico das novas tecnologias, seja pelo reconhecimento dos benefícios das novas tecnologias quanto do reconhecimento da necessidade de novas tecnologias. Desta forma, a aplicação da metodologia busca integrar as duas abordagens - 'technological-push' e 'marketing-pull' - no suporte à decisão da adoção de novas tecnologias.

A seguir, um exemplo de aplicação da metodologia proposta contemplando um método de inter-relacionamento entre as matrizes não utilizado pela literatura pesquisada - a teoria dos conjuntos difusos ("fuzzy set theory") - é apresentado.

\section{Exemplo de Aplicação}

$\mathrm{E}$ ste tópico descreve a aplicação da metodologia de análise estratégica da tecnologia em uma empresa de autopeças, que atende basicamente dois mercados com características distintas: as montadoras (mercado A) e os distribuidores de peças de reposição (mercado B). Os produtos fabricados pela empresa são projetados e especificados pelas montadoras. Os mercados onde ela atua pressionam por reduções de preço ao longo do tempo, exigem altos níveis de qualidade bem como de pontualidade de entrega, além de apresentarem oscilações nãoprevistas na demanda; estas são caracterizadas por pedidos urgentes, e por alterações nas quantidades e nos prazos de entrega dos pedidos previamente colocados. Desta forma, a empresa julga que os critérios competitivos da manufatura relevantes são:

- custo;

- qualidade de conformidade;

- pontualidade;

- velocidade de entrega;

- flexibilidade de volume; e

- flexibilidade de entrega.

Por outro lado, considerando que este mercado é extremamente competitivo, a empresa tem adotado uma postura proativa, ou seja, busca identificar novas oportunidades de mercado e de novas tecnologias. Assim, a área comercial prevê que as montadoras continuarão reduzindo o número de fornecedores concomitantemente à expansão do mercado, o que oferece a oportunidade de aumento da demanda dos atuais produtos - além da incorporação de produtos similares - às empresas com desempenhos superiores aos dos concorrentes, particularmente quanto aos critérios custo e pontualidade. Ainda, a área de manufatura pressiona pela decisão de se acelerar a implantação do Sistema da Qualidade QS 9000, bem como de se melhorar o Sistema de PPCP atual - e para tanto, sugere como alternativas a implantação de um novo e mais moderno Sistema ERP integrado a um Sistema de PPCP com Capacidade Finita. E, 
Quadro 2 - A classificação das tecnologias selecionadas no exemplo de aplicação.

\begin{tabular}{|c|l|c|c|c|c|c|}
\cline { 2 - 6 } \multicolumn{1}{c|}{} & Classificação & $\begin{array}{c}\text { Tecnologia } \\
\text { de } \\
\text { Processo }\end{array}$ & $\begin{array}{c}\text { Tecnologia } \\
\text { de } \\
\text { Materiais }\end{array}$ & $\begin{array}{c}\text { Tecnologia } \\
\text { de } \\
\text { Produtos }\end{array}$ & $\begin{array}{c}\text { Tecnologia } \\
\text { de Infor- } \\
\text { mação }\end{array}$ & $\begin{array}{c}\text { Tecnologia } \\
\text { de } \\
\text { Gestão }\end{array}$ \\
\hline T1 & QS 9000 & & & & $\checkmark$ \\
\hline T2 & $\begin{array}{l}\text { Novo Sistema ERP integrado } \\
\text { com um Sistema de PPCP com } \\
\text { Capacidade Finita }\end{array}$ & & & & $\checkmark$ & $\checkmark$ \\
\hline T3 & Novo Sistema ERP & & & & $\checkmark$ & $\checkmark$ \\
\hline T4 & $\begin{array}{l}\text { Sistema de PPCP com } \\
\text { Capacidade Finita integrado ao } \\
\text { Sistema MRP-II atual }\end{array}$ & & & & $\checkmark$ & $\checkmark$ \\
\hline T5 & Nova máquina automatizada & $\checkmark$ & & & & \\
\hline
\end{tabular}

finalizando, a área de engenharia, identificou uma nova máquina automatizada que oferece um potencial de redução de $50 \%$ no tempo de preparação de determinada operação. Desta forma, é possível relacionar as seguintes tecnologias segundo o Quadro 2.

Este exemplo também considera o conceito de áreas de decisão da manufatura e adota as seguintes utilizadas por PEDROSO (1996): capacidade (AD1), instalações industriais (AD2), tecnologia (AD3), integração vertical (AD4), sistema de PPCP (AD5), fluxo de materiais (AD6), relacionamento com os fornecedores (AD7), gerenciamento da qualidade (AD8), organização (AD9), gerenciamento da força de trabalho (AD10), gerenciamento dos produtos (AD11), medidas de desempenho (AD12) e sistemas de informação (AD13).

Para realizar o inter-relacionamento entre os critérios competitivos da manufatura, as áreas de decisão da manufatura $\mathrm{e}$ as alternativas de tecnologia analisadas, o método escolhido foi o da composição de relações difusas, baseado na teoria dos conjuntos difusos (para maiores detalhes sobre o conceito de 'fuzzy set theory', veja, por exemplo, ZADEH, 1965; BELLMAN \& ZADEH, 1970; ZIMMERMANN, 1985;
ZIMMERMANN, 1987; e DUBOIS \& PRADE, 1989). A escolha deste método foi baseada em duas considerações: ele permite a determinação dos índices de inter-relação de uma maneira intuitiva e a forma de cálculo é relativamente simples em comparação aos demais métodos citados no item 5 deste trabalho. Este método considera que:

se A é uma relação difusa definida em $\mathrm{X}$ e $\mathrm{Y}$

e B é uma relação difusa definida em $\mathrm{Y}$ e $\mathrm{Z}$ então a composição de $\mathrm{A}$ em $\mathrm{B}$ é obtida pela seguinte fórmula:

$$
\begin{aligned}
\operatorname{AoB}<=>\mu_{\mathrm{AoB}}(\mathrm{x}, \mathrm{y})= & \\
=\underset{y}{\max } \min & \left\{\mu_{\mathrm{A}}(\mathrm{x}, \mathrm{y}), \mu_{\mathrm{B}}(\mathrm{y}, \mathrm{z})\right\}= \\
= & \vee\left\{\mu_{\mathrm{A}}(\mathrm{x}, \mathrm{y}) \wedge \mu_{\mathrm{B}}(\mathrm{y}, \mathrm{z})\right\} \\
\mathrm{y} &
\end{aligned}
$$

O critério adotado contempla o potencial de melhoria em relação ao desempenho atual. Para tanto, os índices de inter-relação foram definidos de acordo com a escala apresentada no Quadro 3.

Desta forma, as seguintes perguntas foram realizadas: 
Quadro 3 - Os índices de inter-relação considerados no exemplo de aplicação.

\begin{tabular}{|c|l|}
\hline ÍNDICE & \multicolumn{1}{c|}{ INTERPRETAÇ̃̃O } \\
\hline 1,0 & proporciona uma melhoria excepcionalmente forte no desempenho \\
\hline 0,9 & proporciona uma melhoria muito forte no desempenho \\
\hline 0,7 & proporciona uma melhoria forte no desempenho \\
\hline 0,5 & proporciona uma melhoria moderada no desempenho \\
\hline 0,3 & proporciona uma melhoria fraca no desempenho \\
\hline 0,1 & proporciona uma melhoria muito fraca no desempenho \\
\hline 0,0 & não proporciona melhoria no desempenho \\
\hline 0,$8 ; 0,6 ; 0,4 ; 0,2$ & valores intermediários entre os índices adjacentes \\
\hline
\end{tabular}

- Qual a melhoria esperada em cada um dos critérios competitivos da manufatura ao se melhorar cada uma das áreas de decisão da manufatura?

- Qual a melhoria esperada em cada área de decisão da manufatura ao se implantar cada uma das tecnologias - ou dos conjuntos de tecnologias - analisados?

A cada uma destas perguntas correspondem, respectivamente, as matrizes de inter-relação áreas de decisão da manufatura vs. tecnologias alternativas e critérios competitivos da manufatura vs. áreas de decisão da manufatura. Os resultados são apresentados, respectivamente, nas Tabelas 1 e 2.

A partir destas duas matrizes e utilizando o método da composição de relações difusas, obtém-se como resultado a matriz de interrelação critérios competitivos da manufatura vs. tecnologias alternativas. Esta é apresentada na Tabela 3.

A análise desta última matriz suportou a decisão de investimentos e priorização das alternativas tecnológicas previamente eleitas. Assim, a interpretação desta matriz mostra que a adoção das tecnologias T2 (novo Sistema ERP integrado com um Sistema de PPCP com Capacidade Finita) e T4 (Sistema de PPCP com Capacidade Finita integrado com o Sistema MRP-II atual) podem melhorar consideravelmente o desempenho da manufatura em custo, pontualidade, velocidade de entrega, flexibilidade de volume e flexibilidade de entrega. Como a empresa decidiu priorizar a melhoria do desempenho em custo e pontualidade principalmente para atender o mercado das montadoras $-\mathrm{e}$ havia restrições tanto financeiras quanto de disponibilidade dos recursos humanos, ela decidiu:

- implantar inicialmente um Sistema de PPCP com Capacidade Finita (T4);

- continuar a implantação da QS 9000 (T1) na velocidade atual porque, embora esta tecnologia não resulte em melhorias consideráveis no desempenho da manufatura, é um fator qualificador para a continuidade do fornecimento às montadoras; $\mathrm{e}$

- analisar novamente a adoção de novas tecnologias após a implantação do Sistema de PPCP com Capacidade Finita, ou se as restrições financeiras e de recursos humanos forem relaxadas.

\section{Conclusões}

A consideração das três questões - a conceituação da tecnologia, os critérios competitivos da manufatura desdobrados e as abordagens 'technological-push' e 'marketingpull' na decisão da adoção de novas tecnologias - resultou numa metodologia de análise estratégica da tecnologia com uma abordagem contingencial. Assim, a escolha dos critérios competitivos relevantes, a definição das áreas de negócios da empresa - ou das áreas de decisão da manufatura -, bem como a consideração das 
Tabela 1 - A matriz de inter-relação áreas de decisão da manufatura vs. tecnologias alternativas.

\begin{tabular}{|c|c|c|c|c|c|c|c|c|c|c|c|c|c|}
\cline { 2 - 14 } \multicolumn{1}{c|}{} & AD1 & AD2 & AD3 & AD4 & AD5 & AD6 & AD7 & AD8 & AD9 & AD10 & AD11 & AD12 & AD13 \\
\hline T1 & 0,1 & 0,2 & 0,0 & 0,0 & 0,3 & 0,2 & 0,3 & 0,4 & 0,2 & 0,2 & 0,0 & 0,0 & 0,1 \\
\hline T2 & 1,0 & 0,2 & 0,0 & 0,0 & 1,0 & 0,3 & 0,5 & 0,2 & 0,2 & 0,0 & 0,0 & 0,2 & 0,5 \\
\hline T3 & 0,5 & 0,2 & 0,0 & 0,0 & 0,5 & 0,3 & 0,3 & 0,2 & 0,2 & 0,0 & 0,0 & 0,2 & 0,4 \\
\hline T4 & 0,8 & 0,2 & 0,0 & 0,0 & 0,9 & 0,0 & 0,4 & 0,0 & 0,1 & 0,0 & 0,0 & 0,0 & 0,4 \\
\hline T5 & 0,0 & 0,0 & 0,5 & 0,0 & 0,0 & 0,0 & 0,0 & 0,0 & 0,0 & 0,0 & 0,0 & 0,0 & 0,0 \\
\hline
\end{tabular}

Tabela 2 - A matriz de inter-relação critérios competitivos da manufatura vs. áreas de decisão da manufatura.

\begin{tabular}{|c|c|c|c|c|c|c|}
\cline { 2 - 7 } \multicolumn{1}{c|}{} & custo & $\begin{array}{c}\text { qualidade de } \\
\text { conformidade }\end{array}$ & pontualidade & $\begin{array}{c}\text { velocidade de } \\
\text { entrega }\end{array}$ & $\begin{array}{c}\text { flexibilidade de } \\
\text { volume }\end{array}$ & $\begin{array}{c}\text { flexibilidade de } \\
\text { entrega }\end{array}$ \\
\hline AD1 & 0,7 & 0,2 & 0,5 & 0,8 & 1,0 & 0,3 \\
\hline AD2 & 0,3 & 0,1 & 0,2 & 0,3 & 0,3 & 0,3 \\
\hline AD3 & 0,5 & 0,4 & 0,3 & 0,2 & 0,1 & 0,1 \\
\hline AD4 & 0,3 & 0,2 & 0,4 & 0,4 & 0,2 & 0,4 \\
\hline AD5 & 0,8 & 0,1 & 0,7 & 0,7 & 0,8 & 0,9 \\
\hline AD6 & 0,1 & 0,1 & 0,4 & 0,4 & 0,1 & 0,1 \\
\hline AD7 & 0,3 & 0,3 & 0,4 & 0,4 & 0,1 & 0,4 \\
\hline AD8 & 0,3 & 0,4 & 0,2 & 0,1 & 0,0 & 0,1 \\
\hline AD9 & 0,2 & 0,3 & 0,4 & 0,2 & 0,3 & 0,3 \\
\hline AD10 & 0,5 & 0,4 & 0,2 & 0,1 & 0,3 & 0,1 \\
\hline AD11 & 0,0 & 0,0 & 0,0 & 0,0 & 0,0 & 0,0 \\
\hline AD12 & 0,2 & 0,3 & 0,3 & 0,1 & 0,0 & 0,0 \\
\hline AD13 & 0,2 & 0,2 & 0,7 & 0,5 & 0,2 & 0,5 \\
\hline
\end{tabular}

Tabela 3 - A matriz de inter-relação critérios competitivos da manufatura vs. tecnologias alternativas.

\begin{tabular}{|c|c|c|c|c|c|c|}
\cline { 2 - 7 } \multicolumn{1}{c|}{} & custo & $\begin{array}{c}\text { qualidade de } \\
\text { conformidade }\end{array}$ & pontualidade & $\begin{array}{c}\text { velocidade de } \\
\text { entrega }\end{array}$ & $\begin{array}{c}\text { flexibilidade de } \\
\text { volume }\end{array}$ & $\begin{array}{c}\text { flexibilidade de } \\
\text { entrega }\end{array}$ \\
\hline $\mathrm{T} 1$ & 0,3 & 0,4 & 0,3 & 0,3 & 0,3 & 0,3 \\
\hline $\mathrm{T} 2$ & 0,8 & 0,3 & 0,7 & 0,8 & 1,0 & 0,9 \\
\hline $\mathrm{T} 3$ & 0,5 & 0,3 & 0,5 & 0,5 & 0,5 & 0,5 \\
\hline $\mathrm{T} 4$ & 0,8 & 0,3 & 0,7 & 0,8 & 0,8 & 0,9 \\
\hline $\mathrm{T} 5$ & 0,5 & 0,4 & 0,4 & 0,2 & 0,1 & 0,1 \\
\hline
\end{tabular}

posturas proativa e reativa por parte das empresas permite a aplicação da metodologia em ambientes empresariais diversificados.

A aplicação da metodologia deve ser inserida no desenvolvimento da estratégia de manufatura das empresas. Algumas decisões desta precedem a sua utilização - como, por exemplo, quais são os critérios competitivos qualificadores e ganhadores de pedido (HILL, 1995) e em quais critérios a empresa deve priorizar os esforços para ser mais competitiva (SLACK, 1993) - bem como direcionam a análise da matriz critérios competitivos da manufatura vs. tecnologias alternativas.

Deve-se ainda considerar que o resultado da matriz não é a tomada de decisão propriamente 
dita; na realidade, ela é uma ferramenta de suporte à decisão da adoção de novas tecnologias. No exemplo de aplicação apresentado, a decisão contemplou a implantação de um Sistema de PPCP com Capacidade Finita, mesmo considerando que a adoção deste sistema integrado a um novo Sistema ERP poderia trazer melhores resultados. É importante ressaltar que a metodologia proposta focaliza o processo decisório na adoção de novas tecnologias - e este processo é mais importante que os resultados quantitativos que um dado modelo matemático possa apresentar.

Considerando os desdobramentos deste trabalho, uma questão que merece um estudo mais profundo diz respeito aos métodos de interrelação das matrizes propostas, bem como aos critérios para escolher ou justificar a adoção de novas tecnologias. A literatura discute dezenas destes (SWAMIDASS \& WALLER, 1990; NAIK \& CHAKRAVARTY, 1992; CHEN \& SMALL, 1996) sem concluir objetivamente sobre quais são as condições de aplicação de cada um deles; os próprios autores indicam a necessidade de pesquisas adicionais nesta questão. Um método comumente utilizado é o AHP - 'analytical hierarchical process' (KLEINDORFER \& PARTOVI, 1990; ROPER-
LOWE \& SHARP, 1990; MADU \& GEORGANTZAS, 1991; MOHANTY \& VENKATARAMAN, 1993; O'BRIEN \& SMITH, 1993; KUEI et al., 1994; ALBAYRAKOGLU, 1996); embora os cálculos matemáticos sejam relativamente complexos, o seu uso na prática pode ser viabilizado por 'software' tais como o Expert Choice (KUEI et al., 1994). Uma importante discussão na utilização deste método - e também válida para o exemplo de aplicação apresentado - contempla a questão da interdependência entre as variáveis, uma vez que o método AHP considera como variáveis independentes aquelas que estão no mesmo nível hierárquico (ROPER-LOWE \& SHARP, 1990). Neste caso, as possíveis sinergias entre tecnologias alternativas ou entre os critérios competitivos da manufatura não são consideradas. Uma maneira de minimizar a dependência entre tecnologias alternativas é contemplar conjuntos de tecnologias como uma variável - no exemplo de aplicação, este é o caso das combinações entre o Sistema de PPCP com Capacidade Finita e o Sistema ERP. Porém, como considerar as relações de reforço (SLACK, 1993) entre os critérios competitivos da manufatura? Esta discussão reforça e motiva a continuidade de pesquisas nesta área.

\section{Referências Bibliográficas}

ALBARYAKOGLU, M.M.: "Justification of new manufacturing technology: a strategic approach using the analytic hierarchy process". Production and Inventory Management Journal, v.37, n.1, p.71-6, 1996.

ASHAYERI, J.; TEELEN, A. \& SELEN, W.: "Computer-integrated manufacturing in the chemical industry". Production and Inventory Management Journal, v.37, n.1, p.52-7, 1996.

BELLMANN, R. \& ZADEH, L.: "Decision making”. Management Science, v.17, n.4, p.141-64, 1970.

BERRY, D. \& NAIM, M.M.: "Quantifying the relative improvements of redesign strategies in a P.C. supply chain". International Journal of Production Economics, v.46-47, p.181-96, 1996.
BETZ, F.: Strategic Technology Management. New York, McGraw Hill, 1993.

BOWER, D.J. \& YOUNG, A.: "Infuences of technology strategy in the UK oil and gas-related industry network". In: BENNET, D. \& STEWARD, F. ed. / European Conference on Management of Technology, Birmingham, Jul. 1995. Proceedings. Birmingham, Aston University, p.256-63, 1995.

CHEN, I.J. \& SMALL, M.H.: "Planning for advanced manufacturing technology: a research framework". International Journal of Operations and Production Management, v.16, n.5, p.4-24, 1996.

COOK, W.D. \& KRESS, M.: “A multiple-criteria composite index model for quantitative and qualitative data". European Journal of Operational Research, n.78, p.367-79, 1994. 
CROWE, T.J. \& CHENG, C.-C.: "Using quality function deployment in manufacturing strategic planning". International Journal of Operations and Production Management, v.16, n.4, p.35-48, 1996.

DUBOIS, D. \& PRADE, H.: "Fuzzy sets, probability and measurement". European Journal of Operational Research, n.40, p.135-54, 1989.

GARVIN, D.A.: "Manufacturing strategic planning". California Management Review, v.36, n.3, p.85-106, 1993.

GASPARIKOVA, J.: “Technological strategy - case Slovakia". In: BENNET, D. \& STEWARD, F. ed. / European Conference on Management of Technology, Birmingham, Jul. 1995. Proceedings. Birmingham, Aston University, p.887-89, 1995.

GEHANI, R.R.: "Time-based management of technology: a taxonomic integration of tactical and strategic roles". International Journal of Operations and Production Management, v.15, n.2, p.19-35, 1995.

HIDERFJALL, P.: "Pattens of product innovation in the cardiac pacemaker industry". In: BENNET, D. \& STEWARD, F. ed. / European Conference on Management of Technology, Birmingham, Jul. 1995. Proceedings. Birmingham, Aston University, p.221-29, 1995.

HILL, T.: Manufacturing Strategy: Text and Cases, London, MacMillan Press, 1995.

JOLLY, D. \& HUMBERT, M.: "Playing opposite to power P.C.: the H.P.-Intel alliance". In: BENNET, D. \& STEWARD, F. ed. / European Conference on Management of Technology, Birmingham, Jul. 1995. Proceedings. Birmingham, Aston University, p.136-43, 1995.

\& RAMANI, S.V.: "Technology creation in the biotechnology sectors: the french connection". In: BENNET, D. \& STEWARD, F. ed. / European Conference on Management of Technology, Birmingham, Jul. 1995. Proceedings. Birmingham, Aston University, p.264-71, 1995.

KAKATI, M.: "Strategic evaluation of advanced manufacturing technology". International Journal of Production Economics, v.53, p.141-56, 1997.

KLEINDORFER, P.R. \& PARTOVI, F.Y.: "Integrating manufacturing strategy and technology choice". European Journal of Operational Research, n.47, p.214-24, 1990.
KOHLER, C. \& SCHMIERI, K.: "Computerintegrated manufacturing (CIM) - technological and organizational change in the West Germany capital goods industry". Journal of Manufacturing Systems, v.10, n.1, p.21-31, 1991.

KUEI, C.-H.; LIN, C.; AHETO, J. \& MADU, N.: “A strategic decision model for the selection of advanced technology". International Journal of Production Research, v.32, n.9, p.2117-30, 1994.

LEE, G.L. \& OAKES, I.K.: "The impact of process innovation on supply chain relationships: some smaller firm perspectives". In: BENNET, D. \& STEWARD, F. ed. / European Conference on Management of Technology, Birmingham, Jul. 1995. Proceedings. Birmingham, Aston University, p.161-68, 1995.

MADU, C.N. \& GEORGANTZAS, N.C.: "Strategic thrust of manufacturing automation decisions: a conceptual framework". IIE Transactions, v.23, n.2, p.138-48, 1991.

MITCHELL, G.R.: "Alternative framework for technology strategy". European Journal of Operational Research, n.47, p.153-61, 1990.

MOHANTY, R.P. \& VENKATARAMAN, S.: "Use of the analytic hierarchy process for selecting automated manufacturing systems". International Journal of Operations and Production Management, v.13, n.8, p.45-57, 1993.

MUNRO, H. \& NOORI, H.: "Measuring commitment to new manufacturing technology: integrating technological push and marketing pull concepts". IEEE Transactions on Engineering Management, v.35, n.2, p.63-70, 1988.

NAIK, B. \& CHAKRAVARTY, A.K.: "Strategic acquisition of new manufacturing technology: a review and research framework". International Journal of Production Research, v.30, n.7, p.1575-601, 1992.

NELSON, R.R. (ed.): National Innovation Systems: a Comparative Analysis. New York, Oxford University Press, 1993.

NOORI, H. \& GILLEN, D.: “A performance measuring matrix for capturing the impact of AMT". International Journal of Production Research, v.33, n.7, p.2037-48, 1995.

O'BRIEN, C. \& SMITH, S.J.E.: "Design of the decision process for strategic investment in advanced manufacturing systems". International Journal of Production Economics, v.30-31, p.309-22, 1993. 
PEGELS, C.C. \& THIRUMURTHY, M.V.: "The impact of technology strategy on firm performance". IEEE Transactions on Engineering Management, v.43, n.3, p.246-49, 1996.

PEDROSO, M.C.: MISPEM: Modelo de Integração do Sistema de PPCP à Estratégia de Manufatura. Dissertação de Mestrado, Escola Politécnica, Universidade de São Paulo, São Paulo, 1996.

\& CORRÊA, H.L.: "Sistemas de programação da produção com capacidade finita uma decisão estratégica?" Revista de Administração de Empresas, v.36, n.4, p.60-73, 1996.

REAVILL, L. \& KAMALL, S.: "The technological restructuring of the telecomunications system in Hungary". In: BENNET, D. \& STEWARD, F. ed. / European Conference on Management of Technology, Birmingham, Jul. 1995. Proceedings. Birmingham, Aston University, p.857-64, 1995.

ROPER-LOWE, G.C. \& SHARP, J.A.: "The analytic hierarchy process ans its application to an information technology decision". Journal of Operational Research Society, v.41, n.1, p.49-59, 1990.

SHAW, B.: "The restructuring of the technological innovattion system in the russian aerospace industry". In: BENNET, D. \& STEWARD, F. ed. / European Conference on Management of Technology, Birmingham, Jul. 1995. Proceedings. Birmingham, Aston University, p.865-72, 1995.

SLACK, N.: Vantagem Competitiva em Manufatura: Atingindo Competitividade nas Operações Industriais. São Paulo, Atlas, 1993.
STONEBRAKER, P.W. \& LEONG, G.K.: Operations Strategy: Focusing Competitive Excellence. Needham Heights, Allyn and Bacon, 1994.

SWAMIDASS, P.M. \& WALLER, M.A.: “A classification of approaches to planning and justifying new manufacturin technologies". Journal of Manufacturing Systems, v.9, n.3, p.181-93, 1990.

TONG, X. \& ZHAO, H.: "National science policy and technology development - push or pull: the case of Taiwan and South Korea". In: BENNET, D. \& STEWARD, F. ed. / European Conference on Management of Technology, Birmingham, Jul. 1995. Proceedings. Birmingham, Aston University, p.913-20, 1995.

VASCONCELLOS, E. \& WAACK, R.S.: "Monitoring the 'health' of a technology alliance: framework and application”. In: BENNET, D. \& STEWARD, F. ed. / European Conference on Management of Technology, Birmingham, Jul. 1995. Proceedings. Birmingham, Aston University, p.111-18, 1995.

ZADEH, L.: "Fuzzy sets". Information and Control, v.8, p.338-53, 1965.

ZIMMERMANN, H.J.: Fuzzy Sets and its Applications, Kluwer-Nijhoff, Dordrecht, 1985.

ZIMMERMANN, H.J.: Fuzzy Sets, Decision Making and Expert Systems, Kluwer Academic, Dordrecht, 1987.

\title{
A METHODOLOGY FOR STRATEGIC EVALUATION OF TECHNOLOGY
}

\begin{abstract}
This paper proposes a methodology for the strategic evaluation of technology in manufacturing companies. With this aim, the article discusses a technology concept and taxonomy, and adopts an extended set of manufacturing competitive criteria. On this basis, a methodology of strategic technology analysis is proposed which is based on the relationships between the proposed technology taxonomy and the set of competitive manufacturing criteria adopted. The proposed model also considers the technological-push and marketing-pull approaches as decision drivers for the adoption of new technology. This paper also presents a case study which considers the fuzzy set theory and some conclusions about the proposed methodology.
\end{abstract}

Key words: manufacturing strategy, technology, strategic evaluation of technology, fuzzy set theory. 